\title{
Evaluation and Development of Digital Collaboration Techniques for Interdisciplinary Collaboration
}

\author{
Nicolai BEISHEIM ${ }^{\mathrm{a}, 1}$, Markus KIESEL ${ }^{\mathrm{b}}$, Markus LINDE ${ }^{\mathrm{a}}$ and Tobias OTT ${ }^{\mathrm{a}}$ \\ a Albstadt-Sigmaringen University, Albstadt, Germany \\ ${ }^{\mathrm{b}} \mathrm{CMC}$-Kiesel GmbH, Hechingen, Germany
}

\begin{abstract}
In innovative industrial companies, collaboration techniques and laboratories have been in use for years. What historically started with teleconferencing instead of business trips has now reached the status of normal tools for interdisciplinary cooperation in these companies with advances in technologies such as PCs and data broadband as well as software such as Computer Aided Design (CAD), Virtual Reality (VR) and Augmented Reality (AR). But the collaboration techniques are often installed in extra rooms, e.g. video conferencing facilities and large VR laboratories. Other technologies such as chat programs can be used by any employee directly at his workplace. Without the use of these techniques, the short development times required today in many industrial sectors cannot be achieved and even remote maintenance of machines at the customer's would be impossible for machine manufacturers. Within a research project, digital collaboration techniques are inspected and evaluated regarding their suitability for interdisciplinary cooperation in various applications. Collaboration techniques include special hardware for graphic simulations and head-mounted displays for $360^{\circ}$ visualization of digital objects. Additionally different software for cooperation with graphics, video and conference programs distributed over several locations is tested, evaluated and partly newly developed. The application cases are processes from engineering and for planning of buildings. Based on the requirements determined during the tests, a new user-friendly mobile collaboration environment was developed. This collaboration environment integrates the different technologies of graphic simulation as well as already established methods such as video transmission or video conferencing and chat programs in a suitcase system.
\end{abstract}

Keywords. Digital Collaboration, Matrix Network, Mixed Reality, Remote Maintenance

\section{Introduction}

These days, the working environment is changing quickly, driven by globalization, outsourcing and cooperation within networks. For employees, this means that they have to face an increasing interdisciplinary collaboration of teams around the world. Especially knowledge work requires an effective communication and collaboration to ensure the exchange of information and knowledge. Nowadays, the exchange of

\footnotetext{
${ }^{1}$ Corresponding Author, Mail: beisheim@hs-albsig.de.
} 
information and documents between persons is largely handled via electronic communication and information media, which leads to new challenges concerning not only interdisciplinary communication, but also intercultural communication based on language barriers etc. Conventional collaboration tools like e-mail or videoconference are no longer sufficient, because it is often difficult to explain complex technical content to someone who has a different professional background and possibly also another native language. With the help of modern colaboration tools including graphical simulation, it should become easier to communicate and therefore the productivity of virtual meetings will increase. Meanwhile, there is a wide spectrum of different sorts of collaboration tools, that are supposed to adress these challenges. However, there is no real structure or categorization for digital collaboration tools in the literature.

Firstly, this paper aims to categorize the common tools for digital collaboration and develop a mobile collaboration environment. This environment is an all-in-one device which is suitable for almost every purpose and serves the thin line between offering as many functions as possible and offering a convenient mobility. It addresses the problem that in many cases, especially in the AR/VR field, the corresponding technologies are not available in every office, laboratory or meeting room. The new developed collaboration environment can be conveniently moved into the appropriate room and started up in just a few simple steps. With the help of this, the times with disorder of many unsorted cables at workplace or in meeting rooms (as seen in Figure 1) are over.

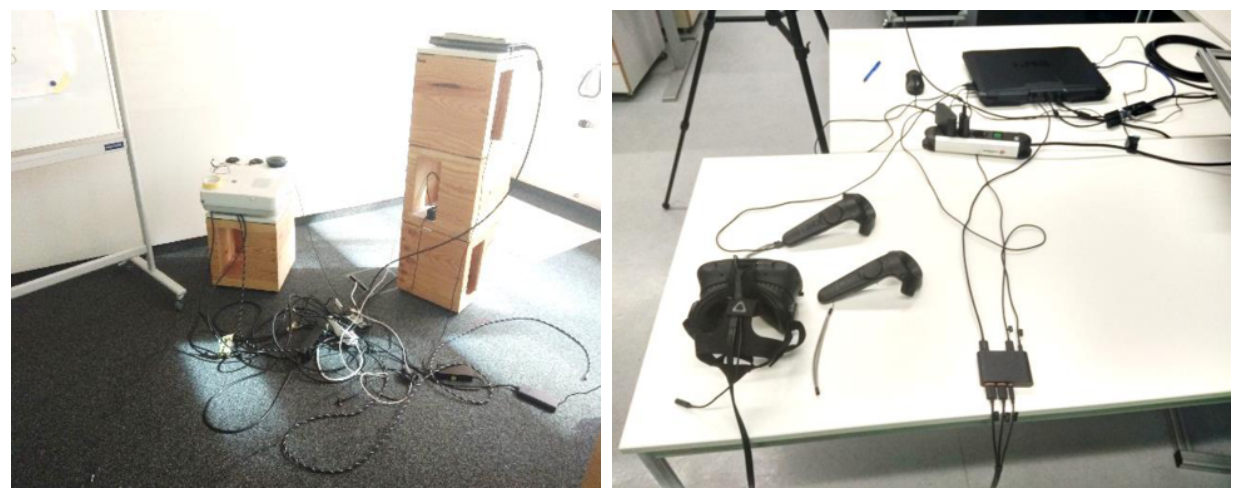

Figure 1. Mess of cables seen on a workshop in Stuttgart and a VR-Laboratory.

If in a collaboration project a large amounts of confidential data must be shared through the internet, the issue of IT Security is gaining in importance as well. Concerning this matter, this paper introducing the Matrix Network as one possible solution. In another chapter an example for collaboration with digital tools the use of Mixed Reality in the field of remote maintenance is given.

\section{Methods and technics for digital collaboration}

A study from the McKinsey Institute has confirmed, that online collaboration tools can improve the employee's productiveness up to $20-25 \%$. [1]

There is a large number of software solutions for digital collaboration but there is no real categorization or struturce in literature so far. The fast moving nature of the IT 
field makes this further complicated by the fact that the new software solutions or plugins are created or published almost on a daily basis.

The website www.capterra.com provides an initial overview of all kinds of software solutions on the market. The search for collaboration related software categories is summarized in Table 1.

Table 1. Results of the search for keywords in www.capterra.com (status: March 2019) [2].

\begin{tabular}{ll}
\hline Software Categories & Number of Software Solutions \\
\hline Collaboration Software & 544 \\
Meeting Software & 153 \\
Web Conferencing Software & 134 \\
Social Network Software & 106 \\
Team Communication Software & 66 \\
Internal Communication Software & 53 \\
Unified Communications Software & 43 \\
Employee Communication Tools & 29 \\
Cloud Communication Platform & 25 \\
\hline
\end{tabular}

Multiple nominations of the same software were possible. There are even more similar Software Categories with numerous solutions. On the one hand, this shows that there must be a great demand for such tools. But on the other hand, it also shows that users are faced with a confusing flood of solutions and it is difficult to select the right product according to the respective individual requirements.

However, the above categories do not say anything about the technique being used. By a more detailed examination, these technique can be divided into the following categories:

- Instant messaging

- Voice and video conferencing

- File sharing

- Screen sharing

- Document collaboration

- Knowledge center

- Internal social network tools

- Project management tools

- AR/VR-collaboration

\section{Use cases and their requirements for collaboration}

The requirements for the mobile collaboration environment in this paper are based on the four current use cases from the research project 'Entwicklung einer mobilen Kollaborationsumgebung EMOKO' at the Albstadt-Sigmaringen University.

The first use case (UC 1) is the industry 4.0 demonstrator in the machine hall of the University in Albstadt. This cyber physical system (CPS) consists of industrial 
robots, conveyors, storage systems and an automated guided vehicle system (AGV). This assembly cell serves to teach students the contents and technologies of industry 4.0. The mobile collaboration environment is supposed to support the interdisciplinary involved persons during the commissioning process using VR/AR Simulations and to stream the content directly to the lecture hall or to the internet.

The second use case (UC 2) is in the BIM (Building Information Modeling) sector. The UC 2 evaluates especially the planning of big commercial kitchens, a special field of expertise of one of the professors involved in the research project. Commonly, nonspecialists have their difficulties by reading und understanding construction plans and design drawings. By using construction specific Virtual and Augmented Reality software, even laypersons should be able to examine and compare the different offers during the bidding phase regarding the food preparation process in a commercial kitchen.

In use case 3 (UC 3), the 'laboratory for digitalization in the food industry' is looking for a remote maintenance solution by using VR/AR Tools. It is the objective to evaluate the use of VR and AR technologies to guide a machine operator throughout a repair or maintenance process by getting advice from an expert located somewhere else.

Use case 4 (UC 4) is settled in the research project 'Digitaler Produktlebenszyklus DiP' (information: http://dip.reutlingen-university.de). The collaboration environment here is supposed to improve the communication between the participating scientists from different universities. It solves the communication problems, particularly in the event of technical problems during the virtual commissioning process with graphical simulation.

After a more detailed analysis of these four use cases, their requirements for collaboration tools can be categorized in:

- Instant messaging

- Audio and video conferencing

- Video recording

- Standard presentation technology

- Remote software

- $\quad$ AR/VR (especially with Head Mounted Displays)

\section{Requirements towards a mobile collaboration environment}

In addition to these specific requirements in the use cases, there are several requirements regarding to the mobility of the collaboration environment as well. These general requirements are:

- Good handling regarding the size and weight (transportable suitcase; should fit into the trunk of a standard car)

- $\quad$ Simple set up and commissioning

- Few outside cables to connect

- Low maintenance

- Cost-effective

- Sustainability (selected hardware and software should still be useable in two or more years) 
The development of a new mobile collaboration environment is based on the combined requirements from the four use cases and other general requirements [3]. For the development process of the colaboration rack IT experts colaborate with mechanical designers to create a solution that provides all the Software and Hardware for the colaboration and also make the rack easy to handle and move to different locations. The developed product is a mobile rack solution, divided into three levels. These levels contain a high-end computer, a slide with convenient foam inlays for the HTC Vive and the associated accessories and on the top of the rack is a sound box and a inclinable touchscreen monitor which are covered with a removable top cover (as seen in Figure 2). In case of need the foam inlay for the Head Mounted Display (HMD) can easily be replaced with an inlay for other HMD's like the Oculus Rift or the Meta-2. For an effortless transport in a normal car, the rack can be dismantled into three individual boxes.
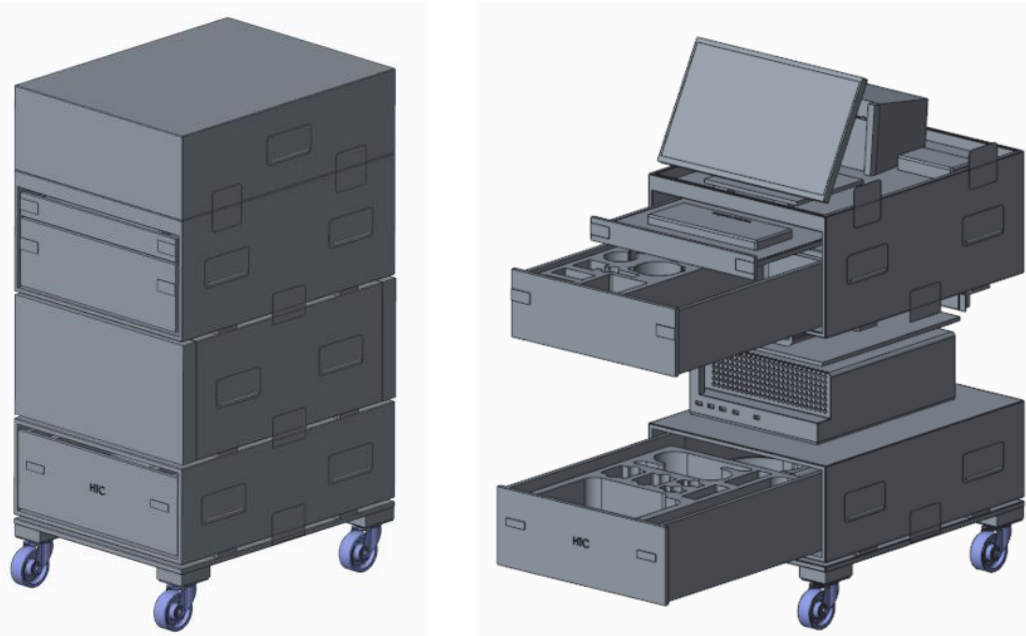

Figure 2. CAD construction of the mobile collaboration environment.

\section{IT Security in collaboration}

Especially when working together in an industrial or research context, there is always some sensitive or confidential information and documents that need to be exchanged. For this purpose, the structure of the IT tool 'Matrix Network' (https://matrix.org/) has turned out to be very useful for a secure communication. It is an open standard for interoperable, decentralized, real-time communication over IP and offers an end-to-end encryption.

To make use of the 'Matrix Network', there are several clients available, based on different programming languages. The Riot Client is the one which offers the most functionality. It works on the common operating systems like Windows, Linux and Mac. There is a desktop and a web application and Riot is even available on Android and iOS. It provides voice and video calls and conferences on all platforms. And most importantly, Riot supports end-to-end encryption which can be chosen to be activate 
for each individual chat. [4] Another advantage compared to many other software products is that there are no license or server costs or other limitations.

For this reasons, the Riot Client is the Standard-Client of choice for the University Albstadt-Sigmaringen, but if cooperating Universitys or Companies require different Software for communication, those can be added, too. Figure 3 shows the IT structure of a network with three universities as an example.

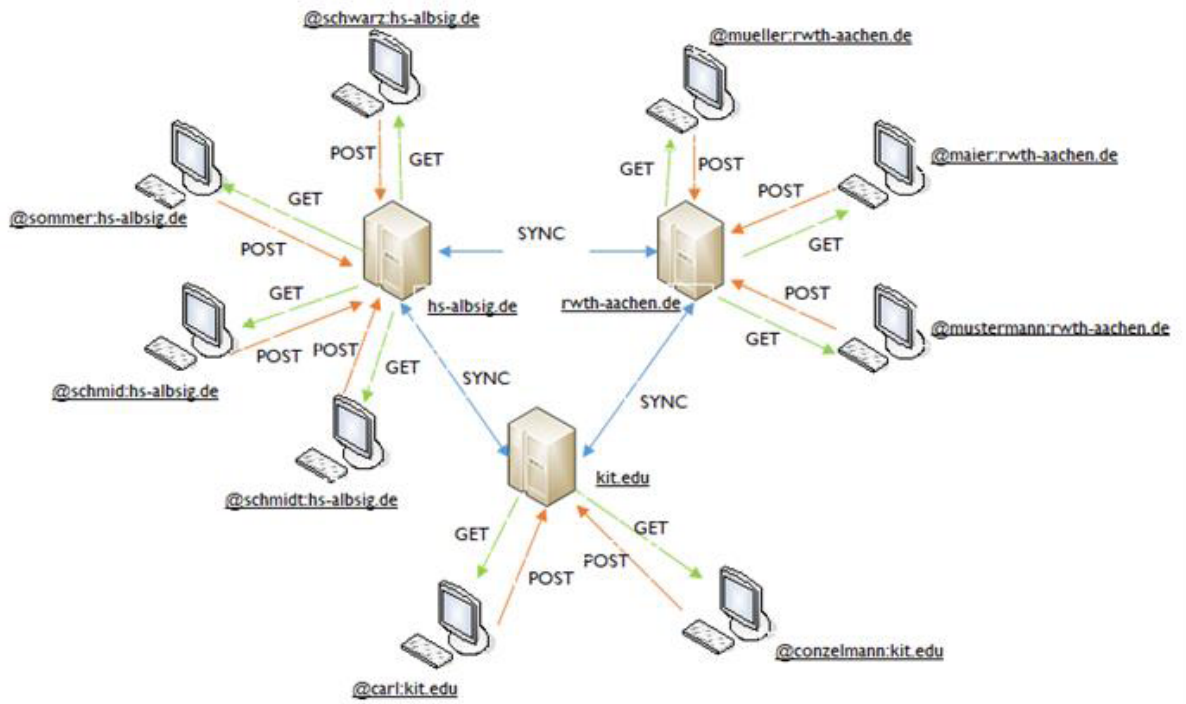

Figure 3. Decentralized structure of the Matrix Network.

\section{Application for the use of Mixed Reality in remote maintenance}

Nowadays, experts and high-skilled mechanics travel around the world from one location to the next location. This is time consuming, high costing and also brings physical and mental stress for the traveler. The use of instructional videos with Mixed Reality can produce relief. The expert can stay at his office and record step-by-step video guides on a virtual twin of the machine in a greenbox and make this instruction available for the machine operator at the location of the occurring problem.

As an industrial application example, the VR-Software CMC ViewR ${ }^{\circledR}$ is used in combination with the Open Broadcaster Software (OBS) [5]. The ViewR Software creates two virtual video signals from the same point of view (foreground and background). These virtual cameras must be positioned at the same position as the real life recording camera.

By cutting the Virtual Reality into two layers, it is possible to immerse into the Virtual Reality and getting obscured by virtual objects (as seen on Figure 4). The variable positions of the cutting plane, which is dividing the Virtual Reality into a foreground and a background scene, is calculated from the live position of the tracked Head Mounted Display (HMD).

OBS combines the video signals of the two virtual cameras and the third signal from the life camera and is able to stream the result to the internet. A chroma key filter 
on the live video record allows to suppress the green background and replace it with the virtual camera videos.

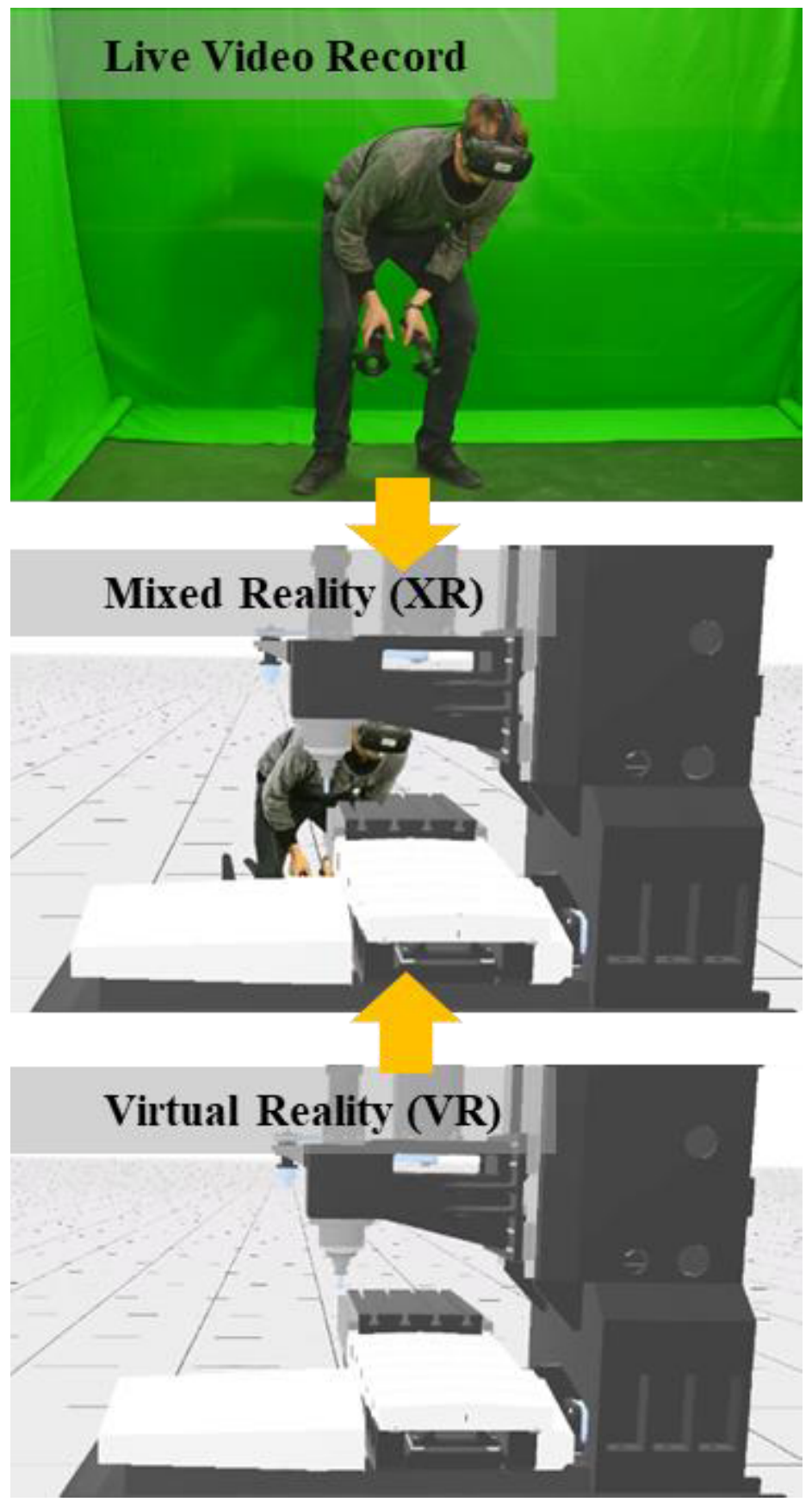

Figure 4. Set Up for Mixed Reality with GreenBox.

The recorded instructional videos can either be streamed as a live session directly to the machine operator or can also be stored in a database as 'how-to-guides' to help 
with common problems in the future. All required hardware and software for this kind of application are included in the new developed mobile collaboration rack, so it is very easy to set up a session for remote maintenance, even for users, that are not extremely familiar with it.

\section{Conclusion}

There are hundreds of software solutions for all sorts of digital collaboration. It is very difficult to define and categorize all of them exactly, since most of them provide more than one of the needed functions at the same time e.g. chatting, video conferencing, file sharing and screen sharing.

The developed mobile environment has to prove itself in the daily work of the different use cases. In combination with the open source Riot Client, the decentralized Matrix Network offers an open standard with plenty of possibilities for interdisciplinary collaboration in research projects and other fields.

The use of Augmented and Virtual Reality technologies has enormous potential in the field of interdisciplinary collaboration between teams around the world. This was evaluated in the research project 'EMOKO' at the University of Albstadt-Sigmaringen. It is easier to discuss technical matters with people that have a different professional background or different native language with the help of graphical simulation, because than it can be explained in Virtual Reality or Augmented Reality Model as graphical IT tools.

\section{References}

[1] M. Chui, J. Manyika, J. Bughin, R. Dobbs, C. Roxburgh, H. Sarrazin, G. Sands, M. Westergren, The social economy: Unlocking value and productivity through social technologies, Accessed: 15.01.2020. [Online]. Available: https:/www.mckinsey.com/industries/technology-media-and-telecommunications/ our-insights/the-social-economy

[2] Capterra Inc., software Categories, www.capterra.com/categories Accessed: 15.01.2020. [Online]. Available: https://www.capterra.com/categories

[3] M. Linde, Entwicklung einer mobilen Kollaborationsumgebung zum Einsatz in Bildungseinrichtungen und Evaluation geeigneter Softwaretools mittels Nutzwertanalyse, master thesis, may 2019, AlbstadtSigmaringen University

[4] The Matrix.org Foundation C.I.C, https://www.matrix.org/, Accessed: 15.01.2020. [Online]. Available: https://matrix.org

[5] Open Broadcaster Software, https://obsproject.com/, Accessed: 15.01.2020. [Online]. Available: https://obsproject.com/ 